dietary anaemia. She previously had a history of vague epigastric pain after meals. Her condition generally deteriorated and nausea and anorexia became predominant symptoms. Four days after admission to a geriatric unit she vomited 70 plastic matrices of her Ferrogradumet $C$ tablets, with immediate relief of her symptoms.

One can only assume that in patients with delayed gastric emptying a situation of pyloric stenosis can be brought about by the administration of this type of tablet. The mechanism is presumably accumulation of the "honeycombed" plastic matrices, through which the active drug, together with a watersoluble channelling agent, passes. In cases in which there is a narrowed or constricted stomach outlet one should perhaps be cautious in the use of such delayed-release iron tablets.

Incidentally, in normal circumstances, the filtration process occurs in the upper small intestine, with the ultimate excretion of the matrix, empty, in the stools.

\section{P CARNE-Ross}

Meopham, Kent

\section{A hazard of apple-eating}

SIR,-An apple a day doesn't always keep the doctor away, as can be learnt from the following case.

A 55-year-old woman was referred to our department for neurological examination. She had a history lasting more than 20 years of attacks of dizziness and syncope, mostly in association with meals. The first time she had fainted was believed to be in connection with a delivery about 25 years previously; this had been regarded as some sort of epileptic attack. However, convulsions had never been observed.

During recent years the attacks had become more alarming, with frequent fainting, principally when swallowing in the sitting position. Further questioning showed that apples and strawberries were most likely to provoke the attacks, which consisted of periods of unconsciousness lasting not longer than 30 seconds, still without convulsions. Previous $x$-ray of the oesophagus was normal. The electrocardiogram (ECG) at rest was normal. Electroencephalography had shown a suspected paroxysmal activity. Complete neurological examination was normal.

Because of the history an ECG was registered while the patient was eating an apple. During the act of swallowing the patient had a feeling of dizziness, and an irregular pulse was palpated, but she did not faint. The ECG showed that the patient had a grade -3 atrioventricular block (see figure) which disappeared spontanously after swallowing had ceased. Repeated experiments with apple-eating gave the same results even when the patient had chewed the pieces carefully. Other fruits, such as oranges and pears, gave no reaction, nor did potatoes or bread and butter. Strawberries could not be tried because of the season.

The patient was given hyoscyamine sulphate in small doses, which resulted in a feeling of well- being and safety and she was able to eat apples again. The only necessary treatment would of course have been to exclude apples from the diet.

This case illustrates how easy it is to mistake Adams-Stokes attacks for some sort of neurological disorder, most frequently epilepsy. A wide survey of the literature on this subject has been published by Johansson. ${ }^{1}$ Our case shows that Adams-Stokes attacks may be provoked by vagal stimuli only in special situations. It would have been tempting to assume that the pieces of apple provoked the attacks by a mechanical effect. However, the attacks were noted even after careful chewing and were not provoked by fairly large lumps of potatoes or bread. It may be speculated whether some sort of chemical stimulation of the oesophagus was also necessary.

\section{ThOMas HaVland} GÖRAN FRITHZ

Department of Medicine,

\section{Central Hospital,} 1 Johansson, B W, American fournal of Cardiology,
1961, 7, 874.

\section{Doctors, contraception, and} sterilisation

SIR,-In reply to Dr P W Lambden's letter (28 August, p 528) I agree that the duty of the family doctor is to give total patient care on the prevention and treatment of disease and injury, which also includes advice on contraception. Of course I am in favour of responsible family planning, but I am concerned about some of the early and late results of introducing either synthetic or natural hormones into a normally functioning feminine physiological cycle.

It is recognised that our business is to see that "the drug does no harm" and "the pill" has been shown to cause venous thrombosis and embolism and, more recently, to be associated with vascular tumours in the liver, one of which has been found to be undergoing malignant change. Knowing this, should we not pause for thought and advise treatment which carries no risk to the patient or her offspring should she, after a space of time, wish to have another child?

As for vasectomy, I regret that Dr Lambden seems to have misunderstood my use of the word "mutilation." Surely the excision of a section of the vasa in a normal healthy male, though expertly and neatly carried out, is a mutilation.

There are other means of contraception than by medical or surgical procedures but demanding responsibility on the part of one or both partners.

Ipswich, Suffolk
National Health Service Consultants' Association

SIR,-The National Health Service Consultants' Association was formed in early 1976 following an enthusiastic response to a letter which appeared in the Lancet $^{1}$

Our basic concern is to save and improve the NHS. We are committed to its basic philosophy of providing medical care to those in need regardless of their social or financial status. Inevitably we are critical of certain aspects of the administration of the NHS, but we are increasingly concerned about the damaging effects on the service (which really means on sick people) of industrial action by doctors in pursuit of their own financial interests. In general we feel that the NHS is best served by a whole-time commitment and that there should be appropriate inducements, financial and otherwise, for the vast majority of consultants to wish to make this commitment. We do not feel that existing bodies such as the BMA and Hospital Consultants and Specialists Association adequately represent this point of view to the DHSS or to the public.

As at least half of consultants are whole-time we feel that there must be many of our colleagues who will be sympathetic with the general tenor of the views outlined above.

The association held its inaugural meeting on 20 May 1976 and since then we have been collecting evidence from our members for submission to the Royal Commission on the NHS as we feel it most important that the whole-timers' view should be adequately represented to this body-certainly the views of those consultants who are fully committed to the NHS seems to have been underrepresented in the disputes about pay-beds and about contracts.

I will be happy to send further information about the association and its aims to those of your readers who might be interested in joining us.

SAM BAXTER

National Health Service Consultants' Association o Department of Psychiatry,

Charing Cross Hospital,
London W6

${ }^{1}$ Noone, P, et al, Lancet, 1976, 1, 141.

\section{Consultants' contract}

SIR,-It is apparent from listening to the debates on consultant contracts that the consultant body does not have a clear idea on which to base plans for a new contract. I would like to remedy this deficit by suggesting a contract which combines the advantages of the whole-time contract with those of the part-time contract and introduces a degree of flexibility which it is obvious that consultants desire.

The basic commitment would be to a number of hours' working time during which the consultant would be available for work in hospital or elsewhere. This would be assessed for each consultant and, for the average wholetime consultant, would probably amount to between 40 and 50 hours if the evidence given to the Owen working party is still applicable. Part-time consultants would be able to contract for a lesser amount of "available working time" (AWT). The lower limit of AWTs might be requested by the employing authority and could reasonably be negotiated between 25 and 30 hours. The upper limit of AWTs could be 